\title{
Amadeus
}

International Multidisciplinary Journal IISSN 2525-8281

DOI: 10.14295/aimj.v4i7.87

\section{Neonatal Brachial Plexus Paralysis: A Discussion on the Light of Literature}

Maria Auxiliadora Ferreira Brito $^{1 ;}$ Modesto Leite Rolim Neto ${ }^{2}$

\begin{abstract}
Neonatal Brachial Plexus Paralysis (PPBN) is defined as a brachial plexus traction or compression injury resulting from dystocic delivery. The incidence of PPBN varies from 0.42 to $5.1 \%$ per 1000 births. The lesion occurs in the expulsive period of childbirth, is most often associated with shoulder dystocia, gestational or pre-gestational diabetes and macrosomic fetuses. The lesion resulting from $\mathrm{C} 5-\mathrm{C} 6$ root involvement is the most frequent, affecting around 50 to $60 \%$ of the cases, and $80 \%$ of these patients have spontaneous recovery. However, around 20 to $30 \%$ of patients may develop persistent deficits, with permanent impacts on upper limb function, compromising the child's development; leading to repercussions for their families and for the health system. PPBN is usually diagnosed by clinical examination and usually does not require imaging studies. Initial treatment is conservative based on functional rehabilitation through physical therapy. Sequential physical examination will indicate patients with no recovery of shoulder movement between 3 and 6 months, who may benefit from surgical treatment. The prognosis of PPBN depends on the level (pre- or postganglionic), the extent, severity of the lesion, the speed of recovery and the quality of initial clinical management.
\end{abstract}

Keywords: Obstetric Paralysis, Brachial plexus, Neonatal

1 School of Medicine, Federal University of Cariri (UFCA), Barbalha, Ceará, Brazil. britomariaauxiliadora@gmail.com;

2 School of Medicine, Federal University of Cariri (UFCA), Barbalha, Ceará, Brazil. Modestorolim@yahoo.com.br. 


\section{Paralisia do Plexo Braquial Neonatal: Uma discussão à Luz da Literatura}

\begin{abstract}
Resumo: A Paralisia Plexo Braquial Neonatal (PPBN) é definida como uma lesão por tração ou compressão do plexo braquial, decorrente de parto distócico. A incidência da PPBN varia 0,42 a $5,1 \%$ por 1000 nascimentos. A lesão ocorre no período expulsivo do parto, é na maioria das vezes associada à distócia de ombro, diabetes gestacional ou prévia à gestação e a fetos macrossômicos. A lesão resultante do comprometimento da raiz C5C6 é a mais frequente atingindo em torno de 50 a $60 \%$ dos casos, sendo que $80 \%$ desses pacientes têm recuperação espontânea. No entanto, em torno de 20 a $30 \%$ dos pacientes podem evoluir com deficits persistentes, com impactos permanentes sobre a função do membro superior, comprometendo o desenvolvimento da criança; levando a repercussões para suas famílias e para o sistema de saúde. A PPBN é geralmente diagnosticada pelo exame clínico e normalmente não requer estudo de imagens. O tratamento inicial é conservador com base na reabilitação funcional através da fisioterapia. O exame físico sequencial indicará os pacientes com ausência de recuperação do movimento do ombro entre o 3 e 6 meses, os quais poderão ser beneficiados com o tratamento cirúrgico. O prognóstico da PPBN depende do nível (pré ou pós- ganglionar), da extensão, da gravidade da lesão, da velocidade da recuperação e da qualidade do manejo clínico inicial.
\end{abstract}

Palavras-Chave: Paralisia obstétrica, Plexo braquial, Neonatal.

\section{Introdução}

A Paralisia Plexo Braquial Neonatal (PPBN) é definida como uma lesão por tração ou compressão do plexo braquial, decorrente de parto distócico (Coroneos, 2017). A lesão do plexo braquial em recém-nascidos que ocorre no período expulsivo do parto, é muitas vezes associada à distócia de ombro, diabetes gestacional ou prévia à gestação e a fetos macrossômicos ou, ainda, a crianças de baixo peso com apresentação pélvica. Em casos raros, pode ocorrer em consequência de fatores intra-uterinos, como útero bicórneo, oligohidrâmnios ou bandas amnióticas (Abid, 2016; Abdouni, 2017).

Um estudo realizado por Chauhan e colaboradores nos EUA em 2014, avaliaram 63 publicações de paralisia braquial neonatal e os resultados apontaram que a incidência da patologia foi semelhante nos EUA e nos outros países, sendo de 1,5 e 1,3/1.000 
nascimentos vivos, respectivamente. Uma publicação europeia mais recentes de Annika (2019), relata que a incidência varia de 0,42 a 5,1/1000 nascidos vivos.

Não há dados sobre a incidência da PPBN no Brasil. No entanto, há uma probabilidade de mais baixas taxas decorrente do maior número de cesarianas no país (Heise, 2015).

Dados da literatura mostram sequelas em 20 a $30 \%$ dos pacientes acometidos, o pode comprometer a qualidade de vida desses pacientes, e trazer implicações significativas para os familiares e para o sistema de saúde (Yau, 2018; Smith, 2018).

A apresentação clínica imediatamente após o parto está associada à severidade da lesão; os recém-nascidos acometidos demonstram paralisia flácida unilateral do membro superior envolvido. O prognóstico da PPBN depende do nível (pré ou pós- ganglionar), da extensão, da gravidade da lesão, da velocidade da recuperação e da qualidade do gerenciamento clínico inicial. A grande maioria dos casos tem prognóstico favorável. Um terço dos casos afeta, adversamente, pacientes pelo resto de suas vidas, comprometendo tanto o movimento quanto a função sensorial no membro superior envolvido (Socolocsky, 2015; Yau, 2018). O manejo adequado e precoce dos casos pode minimizar as potenciais complicações.

O objetivo desse trabalho foi realizar uma revisão sobre a Paralisia do Plexo Braquial Neonatal e, além disso, apresentar uma proposta de atendimento para esses pacientes. Nesse contexto, foi realizada pesquisa bibliográficas usando os termos paralisia braquial neonatal e paralisia obstétrica no PubMed, Web of Science e LILACS de janeiro de 2014 à abril de 2019, sendo priorizados os artigos de revisão. Alguns artigos anteriores ao período foram incluídos pelo impacto dos mesmos.

Tabela 1 - Distribuição dos 17 artigos que compuseram o presente estudo.

\begin{tabular}{|c|c|c|c|}
\hline Autor(es) & Título & $\begin{array}{c}\text { Periódico/Tese/ } \\
\text { Dissertação/Livro }\end{array}$ & Ano \\
\hline Abdouni, Y. A. et al. & $\begin{array}{c}\text { Relação entre a idade e o tipo de paralisia } \\
\text { obstétrica do plexo braquial com o movimento de } \\
\text { pronossupinacão do antebraço. }\end{array}$ & $\begin{array}{l}\text { Revista Brasileira de } \\
\text { Ortopedia, } 52(5): 596- \\
600 .\end{array}$ & 2017 \\
\hline Abid, A. & $\begin{array}{c}\text { Brachial plexus birth palsy: Management during } \\
\text { the first year of life. }\end{array}$ & $\begin{array}{c}\text { Orthopaedics \& } \\
\text { Traumatology: } \\
\text { Surgery \& Research } \\
102 \text { S125-S132. }\end{array}$ & 2016 \\
\hline Alvites, R. et al. & $\begin{array}{l}\text { Peripheral nerve injury and axonotmesis: State of } \\
\text { the art and recent advances. }\end{array}$ & $\begin{array}{l}\text { Cogent Medicine. 5: } \\
1466404 .\end{array}$ & 2018 \\
\hline $\begin{array}{l}\text { Annika, J.; Paul, U. } \\
\text { And Anna-Lena, L. }\end{array}$ & $\begin{array}{l}\text { Obstetric brachial palsy- A prospective, } \\
\text { population-based study of incidency, recovery }\end{array}$ & $\begin{array}{l}\text { European Journal of } \\
\text { Paediatric Neurology }\end{array}$ & 2019 \\
\hline
\end{tabular}




\begin{tabular}{|c|c|c|c|}
\hline & $\begin{array}{c}\text { and long-term residual impairment at } 10 \text { to } 12 \\
\text { years of age. }\end{array}$ & $23,87-93$ & \\
\hline $\begin{array}{l}\text { Blaauw, G. and Muhlig, } \\
\text { R. S. Brith Palsy }\end{array}$ & Obstetric Brachial Plexus Palsy. & $\begin{array}{c}\text { Jornal of Neurology \& } \\
\text { Translational } \\
\text { Neuroscience. 5(1): } \\
1075 .\end{array}$ & 2017 \\
\hline $\begin{array}{l}\text { Borges, André } \\
\text { Varandas. }\end{array}$ & $\begin{array}{c}\text { Tratamento não Cirúrgico nas Lesões do Plexo } \\
\text { Braquial. }\end{array}$ & $\begin{array}{c}\text { Dissertação de } \\
\text { Mestrado Integrado em } \\
\text { Medicina. Instituto de } \\
\text { Ciências Biomédicas } \\
\text { Abel Salazar. } \\
\text { Universidade do Porto. }\end{array}$ & 2016 \\
\hline $\begin{array}{c}\text { Buterbaugh, K.L.; Shah, } \\
\text { A.S. }\end{array}$ & $\begin{array}{l}\text { The natural history and management of brachial } \\
\text { plexus birth palsy. }\end{array}$ & $\begin{array}{l}\text { Current Reviews in } \\
\text { Musculoskeletal } \\
\text { Medicine, 9:418-426. }\end{array}$ & 2016 \\
\hline $\begin{array}{l}\text { Chauhan, S.P. } \\
\text { Blackwell, S. B. and } \\
\text { Anath, C.V. }\end{array}$ & $\begin{array}{l}\text { Neonatal brachial plexus palsy: incidence, } \\
\text { prevalence, and temporal trends. }\end{array}$ & $\begin{array}{c}\text { Seminar in } \\
\text { Perynatology } 38210- \\
218 .\end{array}$ & 2014 \\
\hline $\begin{array}{c}\text { Coroneos C. J; } \\
\text { Voineskos S. H.; } \\
\text { Christakis M. K. et al. }\end{array}$ & $\begin{array}{l}\text { Obstetrical brachial plexus injury (OBPI): } \\
\text { Canada's national clinical practice guideline. }\end{array}$ & BMJ Open, 7:e 014141. & 2017 \\
\hline $\begin{array}{l}\text { Heise, C. O.; Martins, } \\
\text { R.; Siqueira, M. }\end{array}$ & $\begin{array}{c}\text { Neonatal brachial plexus palsy: a permanente } \\
\text { challenge. }\end{array}$ & $\begin{array}{l}\text { Arquivos de } \\
\text { Neuropsiquiatria, } 73 \\
\text { (9): } 803-808 .\end{array}$ & 2015 \\
\hline Kriukova, I.A. et al. & $\begin{array}{l}\text { Development of an algorithm for the treatment of } \\
\text { children with brachial plexus birth palsy in the } \\
\text { first mons of life. }\end{array}$ & $\begin{array}{c}\text { Ортопедия, } \\
\text { травматология и } \\
\text { восстановительная } \\
\text { хирургия детского } \\
\text { возраста. Том } 4 . \\
\text { Выпуск } 1 .\end{array}$ & 2016 \\
\hline M. M. Al-Qattan et al. & $\begin{array}{c}\text { Narakas classification of obstetric plexus palsy } \\
\text { revisited. }\end{array}$ & $\begin{array}{l}\text { The Journal of Hand } \\
\text { Surgery (European } \\
\text { Volume, 34E: } 6: 788- \\
791 .\end{array}$ & 2009 \\
\hline $\begin{array}{l}\text { Raducha, Jeremy. E. et } \\
\text { al. }\end{array}$ & $\begin{array}{c}\text { A review of Brachial Plexus Birth Palsy: Injury } \\
\text { and Rehabilitation. }\end{array}$ & $\begin{array}{l}\text { Rhode Island Medical } \\
\text { Journal, november } \\
\text { p.17-21 }\end{array}$ & 2017 \\
\hline Smith, B.W. et al. & $\begin{array}{c}\text { Un Update on the Management of Neonatal } \\
\text { Brachial Plexus Palsy- Replacing Old Paradigms. } \\
\text { A review. }\end{array}$ & $\begin{array}{l}\text { JAMA Pediatrics. april } \\
30 .\end{array}$ & 2018 \\
\hline Socolovsky, M. et al. & $\begin{array}{l}\text { Obstetric brachial plexus palsy: reviewing the } \\
\text { literature comparing the results of primary versus } \\
\text { secondary surgery. }\end{array}$ & $\begin{array}{l}\text { Child's Nervous } \\
\text { System. March Volume } \\
\text { 32, Issue 3, pp 415-425. }\end{array}$ & 2016 \\
\hline Wilson, T. J. & $\begin{array}{l}\text { Prediction for Surgical Intervencion in Neonatal } \\
\text { Brachial Plexus Palsy. }\end{array}$ & $\begin{array}{c}\text { Neurosurgery. } 82: 335- \\
342\end{array}$ & 2018 \\
\hline Yau, C.W.H.; et al. & $\begin{array}{l}\text { Obstetric brachial plexus injuries (OBPIs): } \\
\text { health-related quality of life in affected adults and } \\
\text { parentes. }\end{array}$ & $\begin{array}{l}\text { Health and Quality of } \\
\text { Life Outcomes; } 16: 212 \text {. }\end{array}$ & 2018 \\
\hline
\end{tabular}

Fonte: Dados da Pesquisa 
Para uma melhor visualização sobre o ano de publicação dos artigos selecionados, observe-se o gráfico a seguir.

Gráfico 1 - Distribuição dos artigos selecionados para o presente estudo

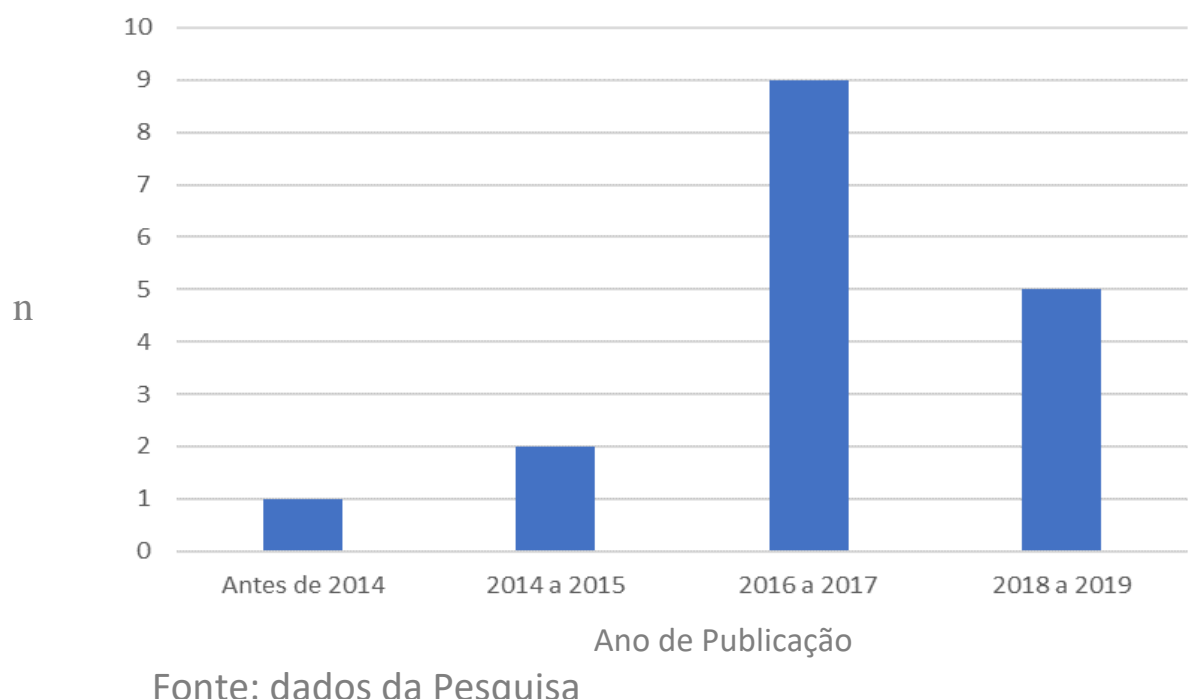

Fonte: dados da Pesquisa

Verificou-se que 2016 a 2017 e, posteriormente em 2018 a 2019 foram os anos com maior número de artigos neste estudo.

\section{Revisão da Literatura}

\section{Anatomia Funcional}

A maioria dos nervos no membro superior tem origem no plexo braquial (PB), fazendo com que esta estrutura tenha uma enorme importância na funcionalidade do membro superior, nomeadamente nos movimentos do ombro, braço e mão, bem como toda a sua sensibilidade. O PB tem origem no pescoço e estende-se à axila, sendo que quase todos os ramos têm origem na axila após esse cruzar a primeira costela. (BORGES, 2016).

O PB é formado pela união dos ramos anteriores dos quatro últimos cervicais (C5C8) e o primeiro nervo torácico (T1), originando a jusante três troncos: o superior (C5 e C6), o médio (C7) e o inferior (C8 e T1). Cada tronco do plexo ramifica-se em divisões anteriores e posteriores na passagem do canal cervicoaxilar posteriormente à clavícula. Assim as divisões anteriores inervam os compartimentos anteriores (flexores) e as posteriores suprem os compartimentos posteriores (extensores) do membro superior. Por 
último, as divisões dos troncos formam três fascículos do plexo, nomeadamente fascículo anterior (divisões anteriores do tronco superior e médio), fascículo medial (divisão anterior do tronco inferior) e o fascículo posterior (Borges, 2016).

A PPBN que se apresenta-se sob formas clínicas distintas, de acordo com a localização e gravidade da lesão neuronal e são as seguintes: Paralisia Alta, ou de ErbDuchenne, Paralisia Média e Paralisia Baixa ou de Klumpke. A paralisia de Erb compromete as raízes C5 e C6, de forma isolada, a paralisia que envolve as raízes C5, C6 e T1 e a paralisia inferior, ou de Klumpke, resulta da lesão das raízes C8 e T1. A lesão de todas as raízes do plexo braquial de C5 a T1 está associada a paralisia total do membro acometido. A paralisia de Erb corresponde a 75\% dos casos (Abid, 2016; Blaauw, 2017).

A classificação proposta por Narakas, se baseia em critérios evolutivos da PPBN, excluindo os casos leves de recuperação em alguns dias, veja tabela 1 abaixo. Considerando que essa classificação faz a estratificação com base no curso clínico das crianças durante as primeiras 8 semanas após o nascimento, não é utilizada para indicação do tratamento cirúrgico (Buterbaugh, 2016; Blaauw, 2017). (Tabela 2).

Tabela 2 - Classificação combinada da Paralisia do Plexo Braquial Perinatal

\begin{tabular}{|c|c|c|c|c|c|}
\hline Grupo & Nome & $\begin{array}{l}\text { Raiz da } \\
\text { lesão }\end{array}$ & $\begin{array}{c}\text { Evolução } \\
\text { da lesão } \\
2^{\mathrm{a}} \text { a } 3^{\mathrm{a}} \\
\text { semanas }\end{array}$ & $\begin{array}{l}\text { Evolução com } \\
2 \text { meses de } \\
\text { idade }\end{array}$ & $\begin{array}{l}\text { Resultado } \\
\text { final }\end{array}$ \\
\hline I & $\begin{array}{l}\text { Paralisia } \\
\text { superior de Erb }\end{array}$ & C5, C6 & $\begin{array}{l}\text { Extensão e } \\
\text { abdução do } \\
\text { ombro }\end{array}$ & $\begin{array}{c}\text { Variável } \\
\text { recuperação da } \\
\text { movimentação } \\
\text { do ombro e } \\
\text { cotovelo }\end{array}$ & $\begin{array}{l}\text { Recuperação } \\
\text { espontânea } \\
>80 \%\end{array}$ \\
\hline IIa & $\begin{array}{l}\text { Paralisia de Erb } \\
\text { com recuperação } \\
\text { da extensão do } \\
\text { punho }\end{array}$ & C5, C6, C7 & $\begin{array}{c}\text { Alterações } \\
\text { do G1 mais } \\
\text { Queda do } \\
\text { punho }\end{array}$ & $\begin{array}{l}\text { Como acima } \\
\text { mais extensão } \\
\text { ativa do punho }\end{array}$ & $\begin{array}{c}\text { Recuperação } \\
\text { espontânea > } \\
60 \%\end{array}$ \\
\hline $\mathrm{IIb}$ & $\begin{array}{l}\text { Paralisia de Erb } \\
\text { sem recuperação } \\
\text { da extensão do } \\
\text { punho }\end{array}$ & C5, C6, C7 & & $\begin{array}{c}\text { Como acima } \\
\text { sem extensão do } \\
\text { punho }\end{array}$ & $\begin{array}{c}\text { Recuperação } \\
\text { espontânea > } \\
60 \%\end{array}$ \\
\hline III & $\begin{array}{l}\text { Paralisia global } \\
\text { não associada } \\
\text { Síndrome de } \\
\text { Horner }\end{array}$ & $\begin{array}{c}\mathrm{C} 5 \text { a C8 } \\
\text { e T1 }\end{array}$ & & $\begin{array}{c}\text { Recuperação } \\
\text { variável do } \\
\text { braço sem } \\
\text { síndrome de } \\
\text { Horner }\end{array}$ & $\begin{array}{c}\text { Recuperação } \\
\text { do braço e } \\
\text { cotovelo 30- } \\
50 \% \\
\text { Mão normal }\end{array}$ \\
\hline IV & $\begin{array}{lr}\text { Paralisia } & \text { global } \\
\text { associada } & \text { à } \\
\text { Síndrome } & \text { de } \\
\text { Horner } & \end{array}$ & $\begin{array}{c}\mathrm{C} 5 \text { a C8 } \\
\text { e T1 }\end{array}$ & & $\begin{array}{c}\text { Recuperação } \\
\text { variável do } \\
\text { braço mais } \\
\text { Síndrome de } \\
\text { Horner }\end{array}$ & $\begin{array}{c}\text { Sem cirurgia } \\
\text { severa, } \\
\text { observa-se } \\
\text { déficits } \\
\text { no braço }\end{array}$ \\
\hline
\end{tabular}

Fonte: Classificação de Narakas segundo Al-Qattan e colaboradores, 2009; com modificações. 
A paralisia de tronco superior, corresponde ao tipo I de Narakas, sendo a alteração típica do membro o "sinal de gorjeta do garçom”; na qual o braço está aduzido e em rotação interna; o cotovelo é estendido, e o pulso está flexionado. Entre os achados ao exame físico, pode-se observar: Reflexo de Moro ausente no lado afetado e reflexo de preensão palmar presente, os défices motores incluem: abdução do ombro, rotação externa e flexão do cotovelo. O reflexo do tendão do bíceps está ausente e a sensibilidade dolorosa está preservada. Na lesão do tronco superior e médio C5 a C7, correspondente de Narakas grau II, além dos déficits motores da Paralisia de Erb, a extensão do punho e da mão estão comprometidas. A flexão dos dedos está presente, porém menor do que no membro saudável. Todos os reflexos tendinosos do membro comprometido estão ausentes. A lesão total do plexo (C5-T1) compromete 20\% dos casos. Alguns pacientes podem preservar movimento menor dos dedos e são classificados como Narakas III. Na paralisia de Klumpke há comprometimento das raízes C8 a T1, compreende menos de 2\% dos casos e envolve tipicamente os músculos do antebraço e da mão, um sinal característico é a mão em garras, devido à perda da função do nervo ulnar e dos músculos intrínsecos da mão que inerva (Heise, 2015; Abid, 2016).

A lesão resultante do comprometimento da raiz C5-C6 é a mais frequente atingindo em torno de 50 a $60 \%$ dos casos, sendo que $80 \%$ desses pacientes têm recuperação espontânea (Buterbaugh, 2015).

O lado direito é afetado em dois terços dos casos, devido à apresentação fetal mais comum. Casos bilaterais são vistos em até 5\%, mas são geralmente assimétricos (Heise, 2015; Borges, 2016).

\section{Etiologia}

Os potenciais mecanismos da PPBN compreendem tração, compressão e estiramento. Destes, o estiramento é considerado o mais frequente, com lesão predominante do tronco superior (Borges, 2016).

A etiologia, atribuída a PPBN, está relacionada à flexão/inclinação lateral da cabeça do feto, quando a distócia de ombro impede o parto normal. A tração lateral e para baixo leva ao estiramento do PB comparado à tração axial descendente em que as forças são aplicadas paralelamente à coluna cervicotorácica do feto. A história obstétrica é 
importante para determinar a etiologia, sendo comum em partos demorados e traumáticos. Fatores ante parto podem predispor à paralisia como útero bicórnio e bandas amnióticas (Borges, 2016; Abid, 2016).

O peso ao nascer é o mais importante fator de risco de PPBN, estando claramente associado à distócia de ombro. Peso ao nascer superior a 4,5 $\mathrm{Kg}$ aumenta em 10 vezes o risco de PPBN. Outros fatores de risco como apresentação pélvica, multiparidade, segunda fase do parto prolongada, diabetes gestacional, filho anterior afetado e préeclâmpsia estão associadas à PPBN (Heise, 2015).

\section{Fisiopatologia}

Para determinar a severidade da lesão, é importante distinguir dois tipos de lesão: a lesão pré-ganglionar e a pós-ganglionar. A pré-ganglionar ou por avulsão, localiza-se acima da raiz dorsal. A lesão que compromete de C8 a T1, é considerada grave por não ser passível de correção cirúrgica direta, havendo necessidade de estudo por ressonância magnética ou por mielografia para o planejamento da cirurgia. Já a lesão pós-ganglionar é localizada abaixo da raiz dorsal ganglionar, sendo descritos três tipos por Seddon e Sunderland (apud Abid, 2016): neuropraxia, anoxotmese e neurotmese. Na neuropraxia há um bloqueio temporário da condução por alongamento simples, os axônios mantém a integridade, levando a paralisia que involui espontaneamente em duas a três semanas. A axonotmese é caracterizada por ruptura neuronal parcial com integridade da bainha com preservação do perineuro e epineuro, podendo haver recuperação espontânea; no entanto pode ocorrer comutação. Finalmente, a neurotmese que corresponde a ruptura total do nervo periférico, com degeneração walleriana, que pode evoluir com formação de neuroma. É considerada a lesão neuronal periférica mais severa e, assim como nas avulsões, é incomum haver recuperação espontânea (Abid, 2016; Smith, 2018; Alvites, 2018).

\section{Diagnóstico}

A história materna, o exame físico e o diagnóstico por imagem auxiliam no raciocínio clínico, orientando para a classificação da lesão e na conduta. Informações da 
história obstétrica com foco nos fatores de risco podem evidenciar distórcia de ombro e macrossomia fetal, na maioria das vezes.

No exame físico do recém - nascido com suspeita de PNPB, deve-se observar a posição do membro afetado em repouso, os movimentos espontâneos, os quais dão uma indicação da extensão da lesão, do défice de força; a seguir os reflexos osteotendinosos e os reflexos primitivos são pesquisados. É importante palpar a clavícula e o úmero do RN, fraturas desses ossos podem afetar os movimentos do membro comprometido e ser fator de confusão com paralisia do PB. Nas lesões do tronco superior (C5-C6), o membro afetado apresenta-se em adução e rotação interna do ombro, o cotovelo em extensão, antebraço em pronação, assimetria ao reflexo de Moro e, diminuição dos reflexos tendinosos. Nas lesões do tronco inferior (C8-T1), observa-se mão em garra, antebraço em pronação e a criança não apresenta movimentos ativos. Deve ser investigada, particularmente, a tríade da Síndrome de Horner composta por miose, ptose e enoftalmia, a qual indica severidade da lesão (Raducha, 2017; Smith, 2018).

A mobilização do membro pode ser dolorosa, particularmente na primeira semana de vida, o que confirma a flacidez da monoplegia. Considerando a impossibilidade de avaliar a variação do movimento ativo, utiliza-se o reflexo de Moro para avaliar rotadores externo do ombro; a preensão palmar para flexores dos dedos; o reflexo miotático para observar contração após extensão do músculo; e, a estimulação cutânea em zona oposta ao músculo em questão (Buterbaugh, 2016; Abid, 2016).

\section{Exames complementares}

Na fase inicial, o exame clínico é crucial para avaliar o diagnóstico e a severidade da lesão; não havendo indicação de nenhum exame diagnóstico. A radiografia poderá ser indicada para pesquisa de fraturas associadas como de clavícula e úmero; pequenas fraturas fisárias podem não ser percebidas ao raio x. Radiografia de tórax pode ser solicitada quando houver suspeita de paralisia frênica (Heise, 2015; Abid, 2016).

Ainda não há um exame específico para PPBN, a eletromiografia (EMG), pode ser solicitada. Os resultados da EMG têm alta probabilidade de falso negativos/positivos nos primeiros meses em $10 \%$ dos casos. A ressonância magnética-RNM da coluna cervical é utilizada no pré-operatório, podendo ser detectada pseudomeningocele, sendo fortemente sugestiva de avulsão radicular. Entretanto, sua confiabilidade permanece 
próxima à da mielografia por tomografia computadorizada-TC. A RNM tem a vantagem de ser um exame direto e não invasivo, que é cada vez mais fácil de realizar com sedação simples, enquanto a mielografia por TC sempre requer anestesia geral (Abid, 2016; Smith, 2018).

\section{Diagnóstico Diferencial}

O diagnóstico diferencial principal se faz com a lesão fisária do úmero proximal que pode simular quadro semelhante à BPBP. Essa lesão, responsável pela pseudoparalisia dolorosa unilateral, não é visível na radiografia, pois a epífise ainda não está ossificada. Em caso de dúvida, uma ultrassonogrfia (US) pode ser solicitada. A verificação radiográfica 10-15 dias depois confirma, retrospectivamente, o diagnóstico pela presença de formação de calo ósseo. Outras fraturas como fratura de clavícula ou de diáfise umeral podem ser identificadas, podendo estar isolada ou em associação com Paralisia Plexo Braquial Neonatal (Abid, 2016).

Osteoartrite séptica de ombro, é rara, pode simular PPBN, o contexto clínico é constituído por prematuridade e paciente hospitalizado em ambiente UTI; estando indicado estudo ultrassonográfico. A pseudoparalisia de Parrot encontrada na sífilis congênita cursa com limitação da movimentação ativa e dor à movimentação passiva do membro superior, sendo secundária à metafisite. A história de sífilis materna e a positividade da sorologia orientam o diagnóstico. Doença de Springel - é caracterizada pela ausência de migração caudal da escápula durante a vida embrionária, resultando em malformação da cintura escapular. Ao exame físico observa-se elevação da escápula, com a presença do osso omovertebral - formação óssea ou cartilaginosa - ligando o ângulo súpero-medial da escápula com a coluna vertebral; diferente da PPB, a movimentação ativa do ombro está preservada, porém, limitada (Kriukova, 2016; Abid, 2016).

Outras deformações congênitas, são mais raras e, segundo Abid (2016), são diagnosticadas durante a cirurgia de PPB. 


\section{Progressão e Prognóstico}

A história natural da BPBN ainda é motivo de debate. O viés de seleção, a heterogeneidade das lesões de PB e a diversidade das medidas de avaliação explicam a grande variabilidade desses dados. A maioria dos autores concorda que as lesões de PPBN são transitórias, com 75-95\% dos casos evoluindo para a recuperação completa. Estudos mais recentes relatam uma taxa mais baixa de 66\%, com um déficit residual em 20-30\% e alteração considerável de função em 10-15\% dos casos. O prognóstico final está diretamente relacionado ao tipo de lesão inicial. Embora a extensão das lesões possa ser avaliada clinicamente, atualmente nenhum exame pode especificar o tipo de lesão alongamento simples, ruptura ou avulsão. A avaliação prognóstica ao nascimento baseiase apenas em critérios clínicos (Abid, 2016).

A paralisia total e a presença da síndrome de Horner são os principais achados que anunciam um prognóstico desfavorável. O valor prognóstico da paralisia do diafragma é, no entanto, controverso, sendo frequentemente citado em publicações como um fator de mau prognóstico (Heise, 2015; Abid, 2016).

Após o nascimento, é necessário acompanhar o paciente observando a recuperação do mesmo, com esse objetivo o exame clínico deverá ser feito pelo menos uma vez por mês. A avaliação da recuperação baseia-se no teste do músculo bíceps, que permanece nos primeiros seis meses o indicador do monitoramento mais simples e confiável. Conforme a evolução, obtar-se-á por tratamento conservdor ou de reparação do nervo.

Em suma, a combinação de três fatores - o tipo e a extensão das lesões, a Síndrome de Horner e a velocidade da recuperação, fornecerá a avaliação da progressão do paciente e guiará as indicações terapêuticas.

\section{Seguimento no primeiro ano}

Algorítmos tem sido desenvolvidos para nortear as decisões, entre eles, o proposto pela Universidade de Michigan usa uma combinação de exame físico seriado e eletrodiagnóstico. Caso não haja a recuperação da força do bíceps e da mão contra a gravidade aos 6 meses, considera-se a possibilidade de cirurgia. Os pacientes que não se 
recuperam nos primeiros dois meses, devem ser acompanhados na atenção terciária por equipe interdisciplinar (Wilson, 2018)

Na PPBN ocorre um desequilíbrio muscular entre os agonistas e os antagonistas que podem ser responsáveis pela retração e rigidez articular, com o desenvolvimento a curto prazo das deformidades osteoarticulares. O exemplo típico é encontrado na articulação escapulo-umeral, particularmente na perda parcial da função com recuperação incompleta. A fraqueza do músculo rotador lateral e do abdutor leva ao desequilíbrio muscular, favorecendo os rotatores mediais, resultando em fixação progressiva do ombro em rotação medial (Abid, 2016).

A rigidez na rotação medial do ombro é considerada a consequência mais frequente, podendo aparecer muito cedo e, rapidamente se estabelece pela retração dos tecidos moles periarticulares. É responsável por problemas de crescimento da articulação glenoumeral, levando à retroversão da subluxação glenoidal progressiva da cabeça do úmero. Essas deformidades podem aparecer a partir dos 5 meses de idade, podendo evoluir rapidamente para luxação posterior da cabeça umeral. Nesse contexto, a fisioterapia pode evitar deformidades. A reabilitação funcional é, portanto, crucial e deve ser realizada durante a recuperação neurológica. Seus objetivos são a manutenção da amplitude de movimento articular, a prevenção de retrações e o fortalecimento muscular (Abid, 2016; Borges, 2016).

Conforme proposta de seguimento de Smith (2018), a Figura 1 abaixo sugere condutas de acordo com a evolução do paciente. 
Figura 1: Fluxograma de decisões na Paralisia do Plexo Braquial Neonatal

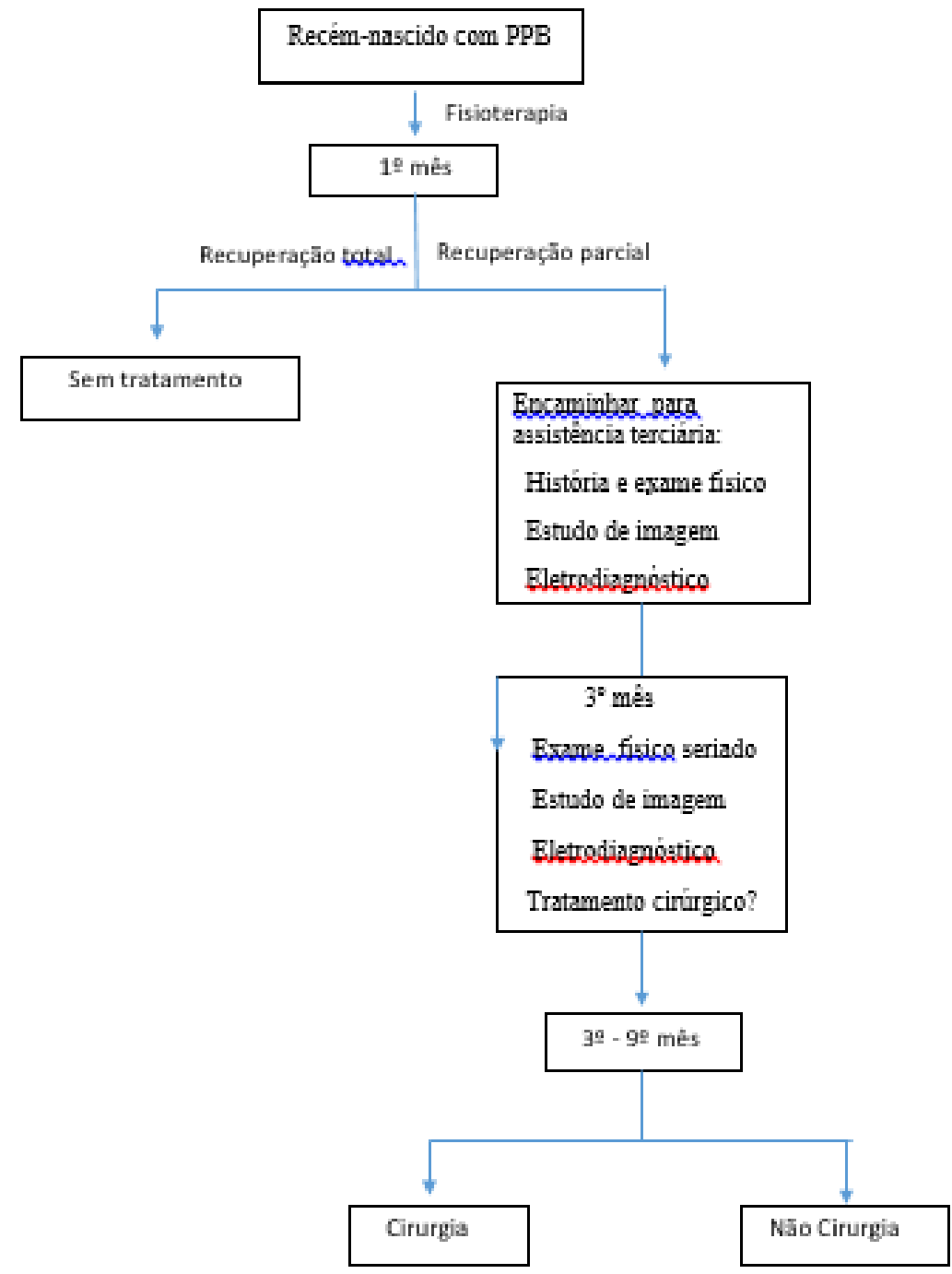

Fonte: Smith (2018), com modificações.

\section{Tratamento}

\section{Tratamento Conservador}

O tratamento inicial é conservador com base na reabilitação funcional Ao contrário do que se pensava, o início precoce da movimento do membro comprometido parece não aumentar o risco de subluxação do ombro. Além disso, a imobilização precoce da região afetada não é indicada e pode ser contra producente para a recuperação do paciente, 
estando indicada em situações específicas. Os objetivos do tratamento fisioterápico incluem manter a amplitude de movimentação e fortalecer os músculos, de forma seletiva, do membro com paralisia. Nesse sentido, a família deve ser treinada para realização de movimentos no domicílio, dando continuidade ao tratamento de fisioterapia (Smith, 2018).

Outras técnicas para tratamento conservador da PPBN, entre elas a estimulação elétrica e dessensibilização, são utilizadas em alguns serviços, ainda que a eficácia seja discutível ( Borges, 2016; Smith, 2018).

\section{Tratamento Cirúrgico}

É consensual que a ausência de recuperação do bíceps até o terceiro mês é indicação para tratamento cirúrgico.

Atualmente, a cirurgia primária do nervo na PPB envolve enxerto de nervo, transferência nervosa ou uma combinação das duas técnicas. Nos casos de avulsão, a técnica de referência indicada consiste de ressecção do neuroma e reparo neurocirúrgico por meio de enxerto de nervo. O resultado do tratamento cirúrgico, pode melhorar de forma significativa a função do membro na PPBN em pacientesadequada selecionado. Dados da literatura, mostram que os resultados são animadores, sendo os eventos adversos raros (Abid, 2016; Smith, 2018).

\section{Conclusão}

A paralisia do plexo braquial no período neonatal representa um desafio para pediatras, cirurgiões, equipe de saúde e para os pais. Requer um diagnóstico, tratamento e reabilitação precoce de modo a proporcionar à criança a melhor recuperação da força muscular e da função sensorial, prevenir as deformidades musculoesqueléticas e integrar o membro superior nos movimentos e atividades adequadas à idade.

Apesar da melhor compreensão da patologia e das opções terapêuticas, não há perspectiva de prevenção num futuro próximo. Seu prognóstico depende em grande parte da gravidade das lesões iniciais, da velocidade de recuperação e da qualidade do tratamento. A paralisia proximal parcial tem um melhor prognóstico do que as paralisias distais; a neuropraxia é melhor que a neurotmese e as lesões pós-ganglionares recuperam melhor que as lesões pré-ganglionares. 
O conhecimento da abordagem inicial do pediatra e de outros membros da equipe de saúde na maternidade são de grande importância para o diagnóstico e terapia com rapidez com vistas a minimizar as complicações. O encaminhamento para centros terciários é crucial, pois uma abordagem multidisciplinar pode ajudar a promover a recuperação e evitar sequelas. Felizmente, há uma alta taxa de recuperação espontânea, mas para os pacientes que não se recuperam espontaneamente, existem opções cirúrgicas e não cirúrgicas para melhorar os resultados funcionais e evitar contraturas devastadoras.

\section{Referências}

Abdouni, Y. A. et al. (2017). Relação entre a idade e o tipo de paralisia obstétrica do plexo braquial com o movimento de pronossupinacão do antebraço. Revista Brasileira de Ortopedia, 52 (5):596-600.

Abid, A. (2016). Brachial plexus birth palsy: Management during the first year of life. Orthopaedics \& Traumatology: Surgery\& Research 102, S125-S132.

Alvites, R. et al. (2018). Peripheral nerve injury and axonotmesis: State of the art and recent advances. Alvites et al., Cogent Medicine. 5: 1466404.

Annika, J.; Paul, U. And Anna-Lena, L. (2019). Obstetric brachial palsy- A prospective, population-based study of incidency, recovery and long-term residual impairment at 10 to 12 years of age. European Journal of Paediatric Neurology 23, 87-93.

Blaauw, G. and Muhlig, R. S. (2017). Brith Palsy ( Obstetric Brachial Plexus Palsy). Jornal of Neurology \& Translational Neuroscience. 5(1): 1075.

Borges, André Varandas. (2016). Tratamento não Cirúrgico nas Lesões do Plexo Braquial. Dissertação de Mestrado Integrado em Medicina. Instituto de Ciências Biomédicas Abel Salazar. Universidade do Porto.

Buterbaugh, K.L.; Shah, A.S. (2016). The natural history and management of brachial plexus birth palsy. Current Reviews in Musculoskeletal Medicine, 9:418-426.

Chauhan, S.P. Blackwell, S. B. and Anath, C.V. (2014). Neonatal brachial plexus palsy: incidence, prevalence, and temporal trends. Seminar in Perynatology 38; 210-218.

Coroneos C. J; Voineskos S. H.; Christakis M. K. et al. (2017). Obstetrical brachial plexus injury (OBPI): Canada's national clinical practice guideline. BMJ Open; 7:e 014141.

Heise, C. O.; Martins, R.; Siqueira, M. (2015). Neonatal brachial plexus palsy: a permanente challenge. Arquivos de Neuropsiquiatria; 73 (9): 803-808. 
Kriukova, I.A. et al. (2016). Development of an algorithm for the treatment of children with brachial plexus birth palsy in the first mons of life. Ортопедия, травматология $и$ восстановительная хирургия детского возраста. Том 4. Выпуск 1.

M. M. Al-Qattan et al. (2009). Narakas classification of obstetric plexus palsy revisited. The Journal of Hand Surgery (European Volume, 34E: 6: 788-791.

Raducha, Jeremy. E. et al. (2017). A review of Brachial Plexus Birth Palsy: Injury and Rehabilitation. Rhode Island Medical Journal, november, p.17-21

Smith, B.W. et al. (2018). Un Update on the Management of Neonatal Brachial Plexus Palsy- Replacing Old Paradigms. A review. JAMA Pediatrics, april 30.

Socolovsky, M. et al. (2016). Obstetric brachial plexus palsy: reviewing the literature comparing the results of primary versus secondary surgery. Child's Nervous System. March, Volume 32, Issue 3, pp 415-425.

Wilson, T. J. (2018). Prediction for Surgical Intervencion in Neonatal Brachial Plexus Palsy. Neurosurgery; 82:335-342.

Yau, C.W.H.; et al. (2018). Obstetric brachial plexus injuries (OBPIs): health-related quality of life in affected adults and parentes. Health and Quality of Life Outcomes; $16: 212$.

\section{How to cite this article (APA format):}

Brito, Maria Auxiliadora Ferreira; Rolim Neto, Modesto Leite Rolim Neto (2019). Neonatal Brachial Plexus Paralysis: A Discussion on the Light of Literature. Am. In. Mult. J., October. (7) 4, 162-177.

Received: $11 / 25 / 2019$

Accepted: 12/02/2019 